Die Berliner Beauftragte für Datenschutz und Informationsfreiheit

\title{
Identitätsdiebstahl darf nicht als allgemeines Geschäftsrisiko hingenommen werden
}

Die Berliner Beauftragte für Datenschutz und Informationsfreiheit beschäftigt sich aktuell verstärkt mit Identitätsdiebstählen im Onlinehandel. Dabei musste die Aufsichtsbehörde bei ihren Prüfungen häufig feststellen, dass Unternehmen nicht genügend Maßnahmen ergreifen, um Identitätsdiebstähle zu verhindern. Allein mit Vornamen, Namen und Geburtsdatum einer anderen Person können Betrüger bei vielen Anbietern die Lieferung von Waren an eine beliebige Anschrift auslösen. Pakete werden mitunter selbst dann auf Rechnung versandt, wenn offensichtliche Unklarheiten hinsichtlich des Bestellers bestehen. Zur Kasse gebeten werden dann zu Unrecht die Opfer des Identitätsdiebstahls, die sich in Folge oft mit Inkassounternehmen und Wirtschaftsauskunfteien auseinandersetzen müssen. Dies ist nicht nur mit großem Recherche- und Zeitaufwand, sondern regelmäßig auch mit erheblichen Kosten verbunden. Zudem droht den Opfern ein negativer Eintrag bei Auskunfteien, der sich nachteilig auf die Bewertung ihrer Zahlungsfähigkeit auswirken und zu Problemen bei Vertragsabschlüssen oder Ratenkäufen führen kann.

Die Berliner Beauftragte für Datenschutz und Informationsfreiheit fordert am 08. September 2017:

- Unternehmen müssen geeignete Methoden zur Identifizierung ihrer Kunden einsetzen. Bei Auffälligkeiten, die auf Betrug hinweisen können, wie etwa einer abweichenden Lieferanschrift, müssen durch die Onlinehändler oder deren Zahlungsdienstleister Kontrollen durchgeführt werden (z. B. eine Melderegisterauskunft oder persönliche Rückfragen).

- Eine Erstbestellung mit einer von der Rechnungsadresse abweichenden Lieferadresse sollte nicht auf Rechnung möglich sein.

- Werden Wirtschaftsauskunfteien im Rahmen der Bestellung angefragt, um die bestellende Person zu identifizieren, müs- sen sie auf Abweichungen, z. B. bei der Adresse, ausdrücklich hinweisen.

- Mahnungen sollten nicht ausschließlich per E-Mail versendet werden. Betrüger geben gern falsche E-Mail-Adressen an, sodass Opfer nicht oder erst sehr spät von dem Identitätsdiebstahl Kenntnis erlangen.

- Wenn bei der Bestellung Unstimmigkeiten hinsichtlich der Identität der bestellenden Person aufgekommen sind, muss dieser Umstand auch einem ggf. später eingeschalteten Inkassounternehmen als Anhaltspunkt für einen möglichen Identitätsdiebstahl mitgeteilt werden.

- Eine negative Meldung an eine Wirtschaftsauskunftei und ein gerichtliches Vorgehen wegen Nichtzahlung gegen die Betroffenen dürfen nur erfolgen, wenn keine Anhaltspunkte für einen Identitätsdiebstahl vorliegen.

- Alle beteiligten Branchen müssen eine effektive und einfache Beschwerdemöglichkeit ohne unnötigen Verwaltungsaufwand schaffen. In jedem Fall muss der Kundenservice für das Thema Identitätsdiebstahl sensibilisiert werden.

- Alle Fälle von Identitätsdiebstahl müssen zur Anzeige gebracht werden. Das ist auch deshalb wichtig, damit die Kriminalstatistik ein aussagekräftiges Bild von dem Phänomen Identitätsdiebstahl geben kann.

Maja Smoltczyk: „Unternehmen dürfen den Schutz von Betroffenen nicht hintenanstellen, um Lieferungen möglichst rasch versenden und Umsatz erzeugen zu können. Wenn es zu einem Identitätsdiebstahl kommt, brauchen die Opfer zudem Unterstützung. Sie dürfen nicht wie potentielle Betrüger behandelt werden. Es wird Zeit, dass der Onlinehandel seiner Verantwortung in diesem Bereich stärker gerecht wird.“

Maja Smoltczyk, die Berliner Beauftragte für Datenschutz und Informationsfreiheit 